University of Nebraska - Lincoln

DigitalCommons@University of Nebraska - Lincoln

Mammalogy Papers: University of Nebraska

State Museum

Museum, University of Nebraska State

5-28-1971

\title{
Systematics of Southern Banner-Tailed Kangaroo Rats of the Dipodomys phillipsii Group
}

Hugh H. Genoways

University of Kansas, h.h.genoways@gmail.com

J. Knox Jones Jr.

University of Kansas

Follow this and additional works at: https://digitalcommons.unl.edu/museummammalogy

Part of the Zoology Commons

Genoways, Hugh H. and Jones, J. Knox Jr., "Systematics of Southern Banner-Tailed Kangaroo Rats of the Dipodomys phillipsii Group" (1971). Mammalogy Papers: University of Nebraska State Museum. 47.

https://digitalcommons.unl.edu/museummammalogy/47

This Article is brought to you for free and open access by the Museum, University of Nebraska State at DigitalCommons@University of Nebraska - Lincoln. It has been accepted for inclusion in Mammalogy Papers: University of Nebraska State Museum by an authorized administrator of DigitalCommons@University of Nebraska Lincoln. 


\title{
J OUR NAL OF MAMMALOGY
}

Published Quarterly by the American Society of Mammalogists

\section{SYSTEMATICS OF SOUTHERN BANNER-TAILED KANGAROO RATS OF THE DIPODOMYS PHILLIPSII GROUP}

\section{Hugh H. Genoways and J. Knox Jones, Jr.}

\begin{abstract}
Both nongeographic and geographic variation was assessed in southern banner-tailed kangaroo rats of the nominal species Dipodomys phillipsii and $D$. ornatus. Univariate and multivariate analyses were employed in consideration of geographic variation. D. ornatus is arranged as a subspecies of D. phillipsii, in which four races (phillipsii, ornatus, perotensis, and oaxacae) are recognized. Some observations on natural history also are included.
\end{abstract}

The southern banner-tailed kangaroo rat, Dipodomys phillipsii, originally was described by Gray (1841:522), based on a specimen from "near Real del Monte," Hidalgo, and is the type species of the genus Dipodomys. The holotype remained the only known specimen of phillipsii until Merriam (1893) reported on material and field observations obtained by E. W. Nelson in the vicinity of Mexico City, and in the states of Tlaxcala, Puebla, and Veracruz. In the following year, Merriam (1894:110-111) named Dipodomys ornatus and Dipodomys perotensis, based on specimens from Berriozábal, Zacatecas, and Perote, Veracruz, respectively, as species that resembled D. phillipsii. Davis (1944:391) reduced perotensis to subspecific status under phillipsii, but ornatus has stood until now in the literature as a distinct species. Finally, Hooper (1947) described Dipodomys phillipsii oaxacae from Teotitlán, Oaxaca, distinguishing it from other known races on the basis of small size and pale coloration. For the currently recognized groups of kangaroo rats, see Lidicker (1960a:134).

Although southern banner-tailed kangaroo rats have been mentioned in various publications dealing with the mammalian faunas of northern and central México, no previous attempt has been made to assess systematically the relationships within this group. Our study is based on 251 specimens, many of them obtained in the last two decades, and includes analysis of both nongeographic and geographic variation in these rats. We have also summarized the available information on natural history.

\section{Methods AND ACKNOWLEDGMENTS}

All measurements recorded beyond are in millimeters; those recorded for crania were taken by means of dial calipers, whereas external dimensions are those recorded on specimen 
labels by field collectors. The measurement depth of cranium was taken using a glass microscope slide as described by Hooper (1952:10). Bacula were measured under a binocular microscope fitted with an ocular micrometer and were drawn with the aid of an ocular grid. Variation in color was assessed using a Photovolt Photoelectric Reflection Meter, Model 610, which gives reflectance readings as a percentage of pure white (see Lawlor, 1965, and Dunnigan, 1967). Readings were taken with red, green, and blue filters in the middorsal region on skins with unworn pelage.

All statistical analyses were performed on the GE 635 computer at The University of Kansas. Univariate analyses were carried out using a program (UNIVAR) written by Power (1970). This program yields standard statistics (mean, range, standard deviation, standard error of the mean, variance, and coefficient of variation) and, when two or more groups are being compared, employs a single-classification analysis of variance ( $F$-test, significance level .05) to test for significant differences between or among the means of the groups (Sokal and Rohlf, 1969). When means were found to be significantly different, the Sums of Squares Simultaneous Test Procedure (SS-STP) was used to determine the maximally nonsignificant subsets.

Multivariate analyses were performed using the NT-SYS programs developed at The University of Kansas by F. J. Rohlf, R. Bartcher, and J. Kishpaugh. Matrices of Pearson's product-moment correlation were computed, and phenetic distance coefficients were derived from standardized character values. Cluster analyses were conducted using UPGMA (unweighted pair-group method using arithmetic averages) on the correlation and distance matrices. A matrix of correlation among characters then was computed and the first three principal components extracted. A three-dimensional stereogram was not prepared from these data because two-dimensional plots were sufficient to depict relationships among the samples studied of the southern banner-tailed kangaroo rat. Discussions of the theory underlying these tests are given by Schnell (1970:42-44) and Atchley (1970:206212); Choate (1970) and Rising (1970) have used these techniques in studies similar to ours.

In order to obtain samples of a sufficient number of specimens for statistical analysis, it was necessary in many cases to group specimens from several geographic localities. In so doing, we attempted to keep the area concerned as small as possible, and we did not include specimens from more than one major physiographic region nor cross any previously recognized taxonomic boundaries. Specimens labeled with reference to the following geographic places comprised the samples used in our analysis (see Fig. 2): sample 1-Durango (Durango, Laguna de Santiaguillo, Morcillo, Vicente Guerrero); sample 2-Durango (La Pila); sample 3-Zacatecas (Hda. San Juan Capistrano, Valparaíso); sample 4-Jalisco (La Mesa María de León); sample 5-Zacatecas (Berriozábal, Fresnillo, Plateado, Trancoso, Villanueva, Zacatecas); sample 6-Aguascalientes (Aguascalientes, Rincón de Romos), and Jalisco (Encarnación de Díaz, Villa Hidalgo); sample 7-Jalisco (Guadalupe de Victoria, Lagos, Matanzas) and San Luis Potosí (Arenal, Bledos); sample 8-Guanajuato (León); sample 9-Querétaro (Tequisquiapam); sample 10-Distrito Federal (Ajusco, Mexico City, Tlalpam) and México (Amecameca, Texcoco); sample 11-Tlaxcala (Huamantla); sample 12-Puebla (Lago Salido) and Veracruz (Guadalupe Victoria, Limón, Perote); sample 13-Puebla (Chalchicomula, Puebla); sample 14-Oaxaca (Teotitlán); sample 15-Puebla (Tehuitzingo).

We gratefully acknowledge the following curators who made material in their institutions available for study (abbreviations of institutional names used in the accounts are given in parentheses): Gorden B. Corbet, British Museum (Natural History) (BM); Robert T. Orr, California Academy of Sciences (CAS); Ticul Alvarez, Escuela Nacional de Ciencias Biológicas, México (ENCB); George H. Lowrey, Jr., Museum of Natural Science, Louisiana State University (LSU); Rollin H. Baker, The Museum, Michigan State University (MSU); Dilford C. Carter, Texas Cooperative Wildlife Collection, Texas A \& M University (TCWC); Emmet T. Hooper, Museum of Zoology, The University of Michigan (UMMZ); Bernardo 
Villa-R., Instituto de Biología, Universidad Nacional Autonóma de México (UNAM); James S. Findley, Museum of Southwestern Biology, University of New Mexico (UNM); Clyde Jones and Charles O. Handley, Jr., United States National Museum (USNM). Specimens in the collections of the Museum of Natural History of The University of Kansas (KU) also were used.

Part of the research on this project was done under the aegis of a contract (DA-49-193MD-2215) with the U. S. Army Medical Research and Development Command. A. Alberto Cadena translated the summary into Spanish.

\section{NONGEOGRAPHIC VARIATION}

Variation with age.-All specimens examined were assigned to one of three age categories and these were studied in order to determine which should be used in taxonomic comparisons. Age categories, modified after Hall and Dale (1939:49-50) and Lidicker (1960a:128), were as follows:

juvenile-deciduous premolars present;

young-permanent premolars present, but slightly worn, and re-entrant enamel angle still present on lingual edge of upper premolars;

adult-re-entrant angle no longer present on upper premolars, occlusal surface of premolars oval in outline.

No "old adults" were found among the available specimens. Juveniles and young were present only in limited numbers and variation with age, therefore, was not tested statistically; it was obvious, however (Table 1), in comparing age classes in a sample from the vicinity of Perote, Veracruz, that individuals classed as juveniles or young were much smaller than adults. In only one measurement, depth of cranium, did young individuals have the same mean as adults, and juveniles averaged smaller than young in all measurements tested. We used only those individuals classed as adults in our analysis of geographic variation.

Juvenile pelage is grayer and darker dorsally than that of adults; furthermore, the individual hairs of juveniles seem to be finer than those of adults and juveniles are not so densely haired. We detected no pelage intermediate between those we have termed "juvenile" and "adult." There may be, however, an earlier pelage, as suggested by Eisenberg (1963:71), not represented in our specimens.

Individual variation.-The three external measurements used in this study had coefficients of variation in a series of adults from Chalchicomula, Puebla (see Table 2), that ranged from 1.8 (length of hind foot for females) to 4.1 (length of tail for males), whereas the six cranial measurements had a range of 1.4 (depth of cranium for males) to 4.1 (length of maxillary toothrow for males and interorbital breadth for females). These values are well within the range of those for rodents of similar size as cited by Long (1968:210-213, 1969:300-301). Males had higher coefficients of variation in four measurements (total length, length of tail, length of hind foot, and greatest length of skull), and females were more variable in the other five. 
TABLE 1.-Variation with age in a sample of Dipodomys phillipsii from vicinities of Perote and Limón, Veracruz.

\begin{tabular}{|c|c|c|c|c|c|c|}
\hline \multirow[b]{2}{*}{ Measurement } & \multicolumn{2}{|r|}{ Juvenile } & \multicolumn{2}{|r|}{ Young } & \multicolumn{2}{|r|}{ Adult } \\
\hline & $N$ & Mean(Range) & $N$ & Mean(Range) & $N$ & Mean(Range) \\
\hline Total length & & $234.3(215.0-244.0)$ & 4 & $266.2(261.0-270.0)$ & 15 & $270.7(254.0-304.0)$ \\
\hline Length of tail & 4 & $144.0(137.0-151.0)$ & 4 & $159.0(154.0-166.0)$ & 15 & $164.8(149.0-190.0)$ \\
\hline $\begin{array}{l}\text { Length of hind } \\
\text { foot }\end{array}$ & 4 & $38.0(34.0-40.0)$ & 4 & $39.8(39.0-40.0)$ & 16 & $40.5(38.0-44.0)$ \\
\hline $\begin{array}{l}\text { Greatest length } \\
\text { of skull }\end{array}$ & 2 & $33.4(32.0-34.8)$ & 4 & $36.4(35.9-36.9)$ & 13 & $37.4(35.3-38.7)$ \\
\hline $\begin{array}{l}\text { Length of max. } \\
\text { toothrow }\end{array}$ & & & 4 & $4.8(4.5-5.1)$ & 17 & $4.9(4.2-6.0)$ \\
\hline $\begin{array}{l}\text { Depth of } \\
\text { cranium }\end{array}$ & 3 & $13.2(12.8-13.4)$ & 4 & $13.4(13.0-13.8)$ & 12 & $13.4(12.9-13.7)$ \\
\hline $\begin{array}{l}\text { Mastoid } \\
\text { breadth }\end{array}$ & 4 & $21.6(21.0-22.5)$ & 4 & $22.8(22.2-23.1)$ & 16 & $23.4(22.7-24.5)$ \\
\hline $\begin{array}{l}\text { Maxillary } \\
\text { breadth }\end{array}$ & 2 & $18.3(17.7-18.8)$ & 4 & $19.8(19.5-20.1)$ & 15 & $20.9(20.0-22.1)$ \\
\hline $\begin{array}{l}\text { Interorbital } \\
\text { constriction }\end{array}$ & 3 & $10.5(9.5-12.0)$ & 4 & $12.6(12.3-12.7)$ & 12 & $13.0(12.3-14.1)$ \\
\hline
\end{tabular}

Color is geographically variable in southern banner-tailed kangaroo rats, but varies to a greater degree among rats from a single locality than do external or cranial measurements. For example, among 14 geographic samples analyzed by us, reflectance of red ranged from 5.5 to 13.1 in coefficient of variation.

Secondary sexual variation.-Analysis of variance was used to test each of nine measurements in a sample from Puebla (Table 2) to determine if the means were significantly different between sexes. Males were found to be significantly longer than females at the .01 level for total length and at the .05 level for length of tail. No significant differences were found between the sexes in the remaining seven measurements, although males averaged slightly larger in all except depth of cranium. We combined values for the two sexes in geographic analysis, but attempted to keep males and females in similar proportions in the samples analyzed.

Seasonal variation.-Molt from one adult pelage to another appears to occur semiannually. The first molt probably begins in late March or early April (9 April earliest date recorded), with all individuals completing the molt by late July (23 July latest date recorded). The second molt begins in midSeptember (14 September earliest date recorded) and is completed by midor late December (14 December latest date recorded). The pattern of molt is similar to that recorded for Perognathus parvus by Speth (1969). Molt begins dorsally just behind the ears and progresses anteriorly and posteriorly as well as ventrally. We detected no seasonal differences in color of pelage in the material at hand. 
TABLE 2.-Secondary sexual variation in adult Dipodomys phillipsii from Chalchicomula, Puebla.

\begin{tabular}{|c|c|c|c|c|}
\hline Sex & $N$ & Mean $\pm 2 \mathrm{SE}$ (Range) & $\mathrm{CV}$ & $F_{s} / F$ \\
\hline \multicolumn{5}{|c|}{ Total length } \\
\hline Male & 17 & $278.4 \pm 4.62(252.0-291.0)$ & 3.4 & $9.84^{* *}$ \\
\hline Female & 13 & $268.2 \pm 4.29(258.0-283.0)$ & 2.9 & 4.20 \\
\hline \multicolumn{5}{|c|}{ Length of tail } \\
\hline Male & 17 & $172.2 \pm 3.46(158.0-182.0)$ & 4.1 & $4.48^{*}$ \\
\hline Female & 13 & $167.0 \pm 3.29(159.0-178.0)$ & 3.6 & 4.20 \\
\hline \multicolumn{5}{|c|}{ Length of hind foot } \\
\hline Male & 19 & $41.3 \pm 0.43(40.0-43.0)$ & 2.3 & $3.33 \mathrm{~ns}$ \\
\hline Female & 13 & $40.8 \pm 0.42(40.0-42.0)$ & 1.8 & 4.17 \\
\hline \multicolumn{5}{|c|}{ Greatest length of skull } \\
\hline Male & 18 & $37.5 \pm 0.44(35.7-38.8)$ & 2.5 & $2.23 \mathrm{~ns}$ \\
\hline Female & 13 & $37.0 \pm 0.44(35.7-38.3)$ & 2.1 & 4.18 \\
\hline \multicolumn{5}{|c|}{ Length of maxillary toothrow } \\
\hline Male & 19 & $4.9 \pm 0.09(4.5-5.3)$ & 4.1 & $3.70 \mathrm{~ns}$ \\
\hline Female & 13 & $4.8 \pm 0.11(4.4-5.2)$ & 4.3 & 4.17 \\
\hline \multicolumn{5}{|c|}{ Depth of cranium } \\
\hline Male & 18 & $13.3 \pm 0.09(12.7-13.5)$ & 1.4 & $1.49 \mathrm{~ns}$ \\
\hline Female & 13 & $13.4 \pm 0.16(12.9-14.0)$ & 2.2 & 4.18 \\
\hline \multicolumn{5}{|c|}{ Mastoid breadth } \\
\hline Male & 19 & $23.1 \pm 0.21(22.3-24.0)$ & 2.0 & $0.12 \mathrm{~ns}$ \\
\hline Female & 13 & $23.0 \pm 0.34(22.0-23.9)$ & 2.6 & 4.17 \\
\hline \multicolumn{5}{|c|}{ Maxillary breadth } \\
\hline Male & 17 & $20.9 \pm 0.23(20.2-21.9)$ & 2.3 & $0.65 \mathrm{~ns}$ \\
\hline Female & 13 & $20.7 \pm 0.46(19.5-22.2)$ & 4.0 & 4.20 \\
\hline \multicolumn{5}{|c|}{ Interorbital constriction } \\
\hline Male & 15 & $12.7 \pm 0.15(12.3-13.3)$ & 2.2 & $0.95 n s$ \\
\hline Female & 10 & $12.5 \pm 0.32(11.8-13.4)$ & 4.1 & 4.28 \\
\hline
\end{tabular}

\section{GeOgraphic Variation}

Specimens of the nominal species Dipodomys ornatus and D. phillipsii were grouped into 14 samples for univariate analysis of geographic variation; a fifteenth sample consisting of a single individual was added for the multivariate analysis (see section on methods and Fig. 2). Samples 1 to 8 are from the geographic range of Dipodomys ornatus (as understood at the outset of this study), whereas samples 10 to 15 are from within the known range of D. phillipsii. Sample 9 is from an area intermediate between the previously known ranges of the two taxa. Table 3 gives standard statistics for external and cranial measurements and for color reflectance of rats from the 15 samples. 


\section{Univariate Analysis}

External measurements.-Total length and length of the tail exhibit little significant geographic variation. The SS-STP yielded only two nonsignificant subsets for both measurements. For total length the first subset is composed of all localities except number 14 and the second subset contained samples $1,2,8,12$, and 14. Examination of means for total length revealed that only two samples, 2 (264.0) and 14 (244.3), yielded values that did not fall between 270 and 280 . The first subset for length of tail again included all samples excepting 14, whereas the second subset included all save 9 ; the mean for specimens comprising sample 14 (157.0) is much smaller than the others, which have values ranging from 162.3 (sample 2) to 176.7 (sample 9). Length of the hind foot exhibited slightly more geographic variation than did other external measurements, being divided into three nonsignificant subsets (Table 4 ): the first contained all samples excepting 5 (central Zacatecas), 2 ( $\mathrm{La}$ Pila, Durango), and 14 (Teotitlán, Oaxaca); sample 3 (western Zacatecas) with the largest mean (42.0) and sample 14 with the smallest (36.3) were the only samples not included in the second subset, whereas the last subset consisted of samples 2, 5, and 14. Inspection of means for length of hind foot revealed no noteworthy breaks in the continuum of variation, except for that between sample 14 and the next nearest mean value, 40.0 (sample 2 ).

Cranial measurements.-Means for greatest length of skull were arranged in five broadly overlapping nonsignificant subsets (Table 4). Specimens from Tlaxcala ( sample 11), Veracruz (12), central Puebla (13), and Querétaro (9) have, on the average, the longest skulls (mean values for greatest length, 37.2 to 37.5). Samples from Zacatecas, Jalisco, Aguascalientes, San Luis Potosí, and Guanajuato ( 3 to 8 ) as well as the sample (10) from Estado de México and the Distrito Federal have means ranging from 36.2 to 36.5 , and the two samples from Durango ( 1 and 2 ) are only slightly smaller ( 35.5 and 35.8). Specimens from Teotitlán, Oaxaca (sample 14), averaged smallest, with a mean length of skull of 34.1.

Length of the maxillary toothrow exhibited little geographic variation, having only two broadly overlapping subsets. The first contained all samples except 13 (central Puebla), 7 (northeastern Jalisco and western San Luis Potosí), and 14 (Teotitlán, Oaxaca), whereas the second contained all samples except the largest individuals, represented by sample 9 (Querétaro). All samples have a mean length of maxillary toothrow in the range of 4.8 to 5.0 with the exception of sample 9 (mean 5.3) and sample 14 (4.4).

Means for depth of cranium were arranged into three nonsignificant subsets. The first contained all samples with the exception of 1,2 , and 14, which have the shallowest crania. Omitted from the second subset are samples 6 (with the deepest cranium) and sample 14 (with the shallowest). The third subset is made up of samples $1,2,4,9,10$, and 14. Visually, the means for this measurement form an unbroken series from 13.0 (value of samples 1 and 2) 
to 13.6 (value of sample 6), except for sample 14, in which the depth of cranium averaged only 12.5 .

Means for mastoid breadth fell into five broadly overlapping nonsignificant subsets. Little can be discerned from examination of these subsets, but the size-order of means is of interest. Samples from Veracruz, Puebla, and Tlaxcala (11 to 13), along with those from Querétaro and Guanajuato (8 and 9), averaged largest in mastoid breadth with values of 22.9 to 23.4. Next in order of decreasing size are samples from Jalisco, Aguascalientes, Zacatecas, and San Luis Potosí (3 to 7) with means of 22.6 to 22.8. The sample from México and Distrito Federal (10) averaged 22.5, followed by two samples from Durango ( 1 and 2) with values of 22.4 and 22.2. Specimens from northern Oaxaca (14) averaged narrowest in mastoid breadth (21.3) among the samples studied.

Means for maxillary breadth formed five broadly overlapping subsets and are of considerable interest when compared with means of mastoid breadth. The average maxillary breadth in samples 8 and 9 (22.5 and 22.0) is large, as were the means for mastoid breadth in these samples. Next in size in maxillary breadth are the samples from Jalisco, Aguascalientes, and San Luis Potosí (means 21.4 to 21.7), and these also grouped together in mastoid breadth. Near the middle of the range of variation for the species is the sample from the vicinity of Mexico City (10) and the two Durangan samples ( 1 and 2), with values of 21.0 to 21.3 ; these three groups had the smallest means, with the exception of sample 14, for mastoid breadth. The three samples from Veracruz, Puebla, and Tlaxcala (11 to 13 ) averaged relatively narrow (mean values 20.8 and 20.9 ), but it is noteworthy that specimens from these samples averaged among the broadest in mastoid breadth. Specimens from Oaxaca (14) averaged narrowest in maxillary breadth (18.8) as they did also in mastoid breadth.

Means for interorbital constriction fell into six broadly overlapping subsets (Table 4). For this measurement, sample 10 had the largest mean although for maxillary breadth it was near the middle of the range of variation and for mastoid breadth it was among the smallest. Samples 8 and 9 again are among the broadest, as they were for the two previous breadth measurements. Specimens from Jalisco, Aguascalientes, Zacatecas, San Luis Potosí, and Durango ( 1 to 7 ) fell in the middle part of the range of variation, as they did for other breadth measurements. Specimens from Veracruz, Puebla, and Tlaxcala (11 to 13) have, on the average, narrow interorbital regions compared with those of other samples, and specimens from northern Oaxaca (14) averaged narrowest in interorbital constriction.

Color reflectance.-The six nonsignificant subsets into which means for red reflectance fell are shown in Table 4. No overall trend in geographic variation is apparent. Specimens from Jalisco and San Luis Potosí (sample 7) and those from northern Oaxaca (14) had the highest reflectance readings. Most of the northern populations had high readings with the exception of samples 
TABLE 3.-Geographic variation in external dimensions, cranial dimensions, and color among 15 samples (see text and Fig. 2) of southern

\begin{tabular}{|c|c|c|c|c|c|c|c|c|c|c|c|c|c|c|c|}
\hline Statistics & $\underset{1}{\text { Sample }}$ & $\underset{2}{\text { Sample }}$ & $\underset{3}{\text { Sample }}$ & $\underset{4}{\text { Sample }}$ & $\underset{5}{\text { Sample }}$ & $\underset{6}{\text { Sample }}$ & $\underset{7}{\text { Sample }}$ & $\underset{8}{\text { Sample }}$ & $\underset{9}{\text { Sample }}$ & $\underset{10}{\text { Sample }}$ & $\underset{11}{\text { Sample }}$ & $\begin{array}{c}\text { Sample } \\
12\end{array}$ & $\underset{13}{\text { Sample }}$ & $\underset{14}{\text { Sample }}$ & $\underset{15}{\text { Sample }}$ \\
\hline \multicolumn{16}{|c|}{ Total length } \\
\hline $\begin{array}{l}N \\
\text { Mean } \\
\text { Minimum } \\
\text { Maximum } \\
2 \mathrm{SE}\end{array}$ & $\begin{array}{c}12 \\
271.5 \\
255.0 \\
288.0 \\
5.82\end{array}$ & $\begin{array}{c}7 \\
264.0 \\
253.0 \\
282.0 \\
8.32\end{array}$ & $\begin{array}{c}7 \\
275.4 \\
252.0 \\
289.0 \\
9.08\end{array}$ & $\begin{array}{c}8 \\
275.8 \\
260.0 \\
302.0 \\
8.92\end{array}$ & $\begin{array}{c}13 \\
274.1 \\
259.0 \\
295.0 \\
5.98\end{array}$ & $\begin{array}{c}9 \\
277.8 \\
264.0 \\
302.0 \\
8.65\end{array}$ & $\begin{array}{c}19 \\
277.6 \\
257.0 \\
297.0 \\
4.75\end{array}$ & $\begin{array}{c}4 \\
273.0 \\
263.0 \\
282.0 \\
7.87\end{array}$ & $\begin{array}{c}11 \\
279.0 \\
264.0 \\
290.0 \\
4.26\end{array}$ & $\begin{array}{c}16 \\
275.3 \\
259.0 \\
285.0 \\
4.71\end{array}$ & $\begin{array}{c}3 \\
279.7 \\
268.0 \\
295.0 \\
16.01\end{array}$ & $\begin{array}{c}12 \\
270.7 \\
254.0 \\
304.0 \\
6.66\end{array}$ & $\begin{array}{c}30 \\
274.0 \\
252.0 \\
291.0 \\
3.67\end{array}$ & $\begin{array}{c}3 \\
244.3 \\
230.0 \\
255.0 \\
14.89\end{array}$ & $\stackrel{1}{252.0}$ \\
\hline \multicolumn{16}{|c|}{ Length of tail } \\
\hline $\begin{array}{l}N \\
\text { Mean } \\
\text { Minimum } \\
\text { Maximum } \\
2 \text { SE }\end{array}$ & $\begin{array}{c}12 \\
169.3 \\
151.0 \\
188.0 \\
6.12\end{array}$ & $\begin{array}{r}7 \\
164.3 \\
155.0 \\
175.0 \\
5.32\end{array}$ & $\begin{array}{c}7 \\
173.3 \\
164.0 \\
183.0 \\
5.84\end{array}$ & $\begin{array}{c}8 \\
168.5 \\
160.0 \\
192.0 \\
8.25\end{array}$ & $\begin{array}{c}13 \\
172.7 \\
162.0 \\
188.0 \\
4.17\end{array}$ & $\begin{array}{c}9 \\
171.6 \\
162.0 \\
190.0 \\
6.76\end{array}$ & $\begin{array}{c}19 \\
172.2 \\
156.0 \\
190.0 \\
4.30\end{array}$ & $\begin{array}{c}4 \\
169.5 \\
167.0 \\
174.0 \\
3.32\end{array}$ & $\begin{array}{c}11 \\
176.7 \\
167.0 \\
185.0 \\
2.66\end{array}$ & $\begin{array}{c}16 \\
172.9 \\
160.0 \\
182.0 \\
3.66\end{array}$ & $\begin{array}{c}3 \\
175.0 \\
165.0 \\
186.0 \\
12.17\end{array}$ & $\begin{array}{c}15 \\
164.8 \\
149.0 \\
190.0 \\
5.51\end{array}$ & $\begin{array}{c}30 \\
169.9 \\
158.0 \\
182.0 \\
2.57\end{array}$ & $\begin{array}{c}3 \\
157.0 \\
150.0 \\
161.0 \\
7.02\end{array}$ & $\stackrel{1}{155.0}$ \\
\hline \multicolumn{16}{|c|}{ Length of hind foot } \\
\hline $\begin{array}{l}N \\
\text { Mean } \\
\text { Minimum } \\
\text { Maximum } \\
2 \text { sE }\end{array}$ & $\begin{array}{l}14 \\
40.3 \\
38.0 \\
42.0 \\
0.57\end{array}$ & $\begin{array}{c}7 \\
40.0 \\
38.0 \\
42.0 \\
1.06\end{array}$ & $\begin{array}{l}10 \\
42.0 \\
39.0 \\
45.0 \\
1.39\end{array}$ & $\begin{array}{c}9 \\
41.1 \\
39.5 \\
44.0 \\
0.84\end{array}$ & $\begin{array}{l}16 \\
40.2 \\
38.0 \\
42.5 \\
0.60\end{array}$ & $\begin{array}{c}9 \\
40.4 \\
39.0 \\
44.5 \\
1.24\end{array}$ & $\begin{array}{l}18 \\
40.5 \\
38.0 \\
42.5 \\
0.54\end{array}$ & $\begin{array}{c}6 \\
41.2 \\
40.0 \\
42.0 \\
0.61\end{array}$ & $\begin{array}{l}11 \\
41.8 \\
41.0 \\
43.0 \\
0.47\end{array}$ & $\begin{array}{l}17 \\
41.2 \\
39.0 \\
43.5 \\
0.57\end{array}$ & $\begin{array}{c}3 \\
41.0 \\
40.0 \\
42.0 \\
1.15\end{array}$ & $\begin{array}{l}16 \\
40.5 \\
38.0 \\
44.0 \\
0.91\end{array}$ & $\begin{array}{l}32 \\
41.1 \\
40.0 \\
43.0 \\
0.32\end{array}$ & $\begin{array}{c}3 \\
36.3 \\
36.0 \\
37.0 \\
0.67\end{array}$ & $\begin{array}{c}1 \\
34.0\end{array}$ \\
\hline \multicolumn{16}{|c|}{ Greatest length of skull } \\
\hline $\begin{array}{l}N \\
\text { Mean } \\
\text { Minimum } \\
\text { Maximum } \\
2 \text { SE }\end{array}$ & $\begin{array}{l}11 \\
35.5 \\
34.4 \\
36.9 \\
0.58\end{array}$ & $\begin{array}{c}8 \\
35.8 \\
34.4 \\
36.8 \\
0.60\end{array}$ & $\begin{array}{l}10 \\
36.5 \\
34.3 \\
37.6 \\
0.76\end{array}$ & $\begin{array}{c}9 \\
36.2 \\
35.0 \\
37.2 \\
0.53\end{array}$ & $\begin{array}{l}14 \\
36.5 \\
35.4 \\
37.7 \\
0.35\end{array}$ & $\begin{array}{c}8 \\
36.3 \\
34.7 \\
38.1 \\
\mathbf{0 . 7 8}\end{array}$ & $\begin{array}{l}18 \\
36.4 \\
34.7 \\
37.7 \\
0.35\end{array}$ & $\begin{array}{c}6 \\
36.4 \\
35.5 \\
37.2 \\
0.56\end{array}$ & $\begin{array}{c}9 \\
37.2 \\
36.2 \\
38.0 \\
0.41\end{array}$ & $\begin{array}{c}16 \\
36.5 \\
35.0 \\
37.5 \\
0.36\end{array}$ & $\begin{array}{c}2 \\
37.5 \\
37.0 \\
38.0 \\
1.00\end{array}$ & $\begin{array}{l}13 \\
37.4 \\
35.3 \\
38.7 \\
\mathbf{0 . 5 1}\end{array}$ & $\begin{array}{l}31 \\
37.3 \\
35.7 \\
38.8 \\
0.32\end{array}$ & $\begin{array}{c}3 \\
34.1 \\
34.0 \\
34.3 \\
0.18\end{array}$ & $\begin{array}{c}1 \\
34.4\end{array}$ \\
\hline \multicolumn{16}{|c|}{ Length of maxillary toothrow } \\
\hline $\begin{array}{l}N \\
\text { Mean } \\
\text { Minimum } \\
\text { Maximum } \\
2 \text { SE }\end{array}$ & $\begin{array}{c}14 \\
5.0 \\
4.2 \\
5.4 \\
0.17\end{array}$ & $\begin{array}{l}8 \\
4.9 \\
4.5 \\
5.3 \\
0.24\end{array}$ & $\begin{array}{l}9 \\
5.0 \\
4.6 \\
5.3 \\
0.18\end{array}$ & $\begin{array}{l}9 \\
4.9 \\
4.4 \\
5.1 \\
0.16\end{array}$ & $\begin{array}{c}17 \\
4.9 \\
4.6 \\
5.4 \\
0.11\end{array}$ & $\begin{array}{l}9 \\
5.0 \\
4.7 \\
5.3 \\
0.15\end{array}$ & $\begin{array}{l}19 \\
4.8 \\
4.3 \\
5.2 \\
0.09\end{array}$ & $\begin{array}{l}6 \\
4.9 \\
4.5 \\
5.1 \\
0.20\end{array}$ & $\begin{array}{c}11 \\
5.3 \\
5.0 \\
5.5 \\
0.12\end{array}$ & $\begin{array}{l}16 \\
5.0 \\
4.7 \\
5.3 \\
0.09\end{array}$ & $\begin{array}{l}3 \\
5.0 \\
4.9 \\
5.0 \\
0.07\end{array}$ & $\begin{array}{l}17 \\
4.9 \\
4.2 \\
6.0 \\
0.21\end{array}$ & $\begin{array}{l}32 \\
4.8 \\
4.4 \\
5.3 \\
0.07\end{array}$ & $\begin{array}{l}3 \\
4.4 \\
4.4 \\
4.5 \\
0.07\end{array}$ & $\begin{array}{l}1 \\
4.4\end{array}$ \\
\hline \multicolumn{16}{|c|}{ Depth of cranium } \\
\hline $\begin{array}{l}N \\
\text { Mean } \\
\text { Minimum } \\
\text { Maximum } \\
2 \text { SE }\end{array}$ & $\begin{array}{l}12 \\
13.0 \\
12.6 \\
13.6 \\
0.15\end{array}$ & $\begin{array}{c}7 \\
13.0 \\
12.5 \\
13.3 \\
0.20\end{array}$ & $\begin{array}{l}10 \\
13.3 \\
12.8 \\
13.6 \\
0.20\end{array}$ & $\begin{array}{c}8 \\
13.2 \\
13.0 \\
13.6 \\
0.14\end{array}$ & $\begin{array}{l}14 \\
13.3 \\
13.0 \\
13.6 \\
0.13\end{array}$ & $\begin{array}{c}8 \\
13.6 \\
12.8 \\
14.4 \\
0.36\end{array}$ & $\begin{array}{l}13 \\
13.2 \\
12.9 \\
13.6 \\
0.13\end{array}$ & $\begin{array}{c}6 \\
13.3 \\
13.1 \\
13.5 \\
0.14\end{array}$ & $\begin{array}{c}7 \\
13.2 \\
12.8 \\
13.6 \\
0.20\end{array}$ & $\begin{array}{l}14 \\
13.2 \\
12.8 \\
13.6 \\
0.13\end{array}$ & $\begin{array}{c}3 \\
13.2 \\
13.1 \\
13.3 \\
0.13\end{array}$ & $\begin{array}{l}12 \\
13.4 \\
12.9 \\
13.7 \\
0.13\end{array}$ & $\begin{array}{l}31 \\
13.3 \\
12.7 \\
14.0 \\
0.09\end{array}$ & $\begin{array}{c}3 \\
12.5 \\
12.4 \\
12.5 \\
0.07\end{array}$ & $\begin{array}{c}1 \\
12.6\end{array}$ \\
\hline
\end{tabular}


TABLE 3. Continued

\begin{tabular}{|c|c|c|c|c|c|c|c|c|c|c|c|c|c|c|c|}
\hline Statistics & $\underset{1}{\text { Sample }}$ & $\underset{2}{\text { Sample }}$ & $\underset{3}{\text { Sample }}$ & $\underset{4}{\text { Sample }}$ & $\underset{5}{\text { Sample }}$ & $\underset{6}{\text { Sample }}$ & $\underset{7}{\text { Sample }}$ & $\underset{8}{\text { Sample }}$ & $\underset{9}{\text { Sample }}$ & $\underset{10}{\text { Sample }}$ & $\underset{11}{\text { Sample }}$ & $\underset{12}{\text { Sample }}$ & $\underset{13}{\text { Sample }}$ & $\underset{14}{\text { Sample }}$ & $\underset{15}{\text { Sample }}$ \\
\hline \multicolumn{16}{|c|}{ Mastoid breadth } \\
\hline $\begin{array}{l}N \\
\text { Mean } \\
\text { Minimum } \\
\text { Maximum } \\
2 \text { SE }\end{array}$ & $\begin{array}{l}13 \\
22.4 \\
21.7 \\
23.9 \\
0.36\end{array}$ & $\begin{array}{c}8 \\
22.2 \\
21.4 \\
22.5 \\
0.28\end{array}$ & $\begin{array}{l}10 \\
22.6 \\
20.8 \\
24.0 \\
0.68\end{array}$ & $\begin{array}{c}9 \\
22.7 \\
22.0 \\
23.6 \\
0.34\end{array}$ & $\begin{array}{l}17 \\
22.8 \\
21.9 \\
23.4 \\
0.20\end{array}$ & $\begin{array}{c}8 \\
22.8 \\
21.5 \\
23.8 \\
0.55\end{array}$ & $\begin{array}{l}19 \\
22.8 \\
21.7 \\
23.3 \\
0.22\end{array}$ & $\begin{array}{c}6 \\
23.3 \\
22.9 \\
23.8 \\
0.30\end{array}$ & $\begin{array}{l}11 \\
23.0 \\
22.3 \\
23.5 \\
0.22\end{array}$ & $\begin{array}{l}15 \\
22.5 \\
22.0 \\
23.0 \\
0.16\end{array}$ & $\begin{array}{c}3 \\
22.9 \\
22.2 \\
23.3 \\
0.73\end{array}$ & $\begin{array}{l}16 \\
23.4 \\
22.7 \\
24.5 \\
0.28\end{array}$ & $\begin{array}{l}32 \\
23.0 \\
22.0 \\
24.0 \\
0.18\end{array}$ & $\begin{array}{c}3 \\
21.3 \\
21.1 \\
21.6 \\
0.31\end{array}$ & $\begin{array}{c}1 \\
21.4\end{array}$ \\
\hline \multicolumn{16}{|c|}{ Maxillary breadth } \\
\hline $\begin{array}{l}N \\
\text { Mean } \\
\text { Minimum } \\
\text { Maximum } \\
2 \text { SE }\end{array}$ & $\begin{array}{l}15 \\
21.1 \\
19.6 \\
21.9 \\
0.32\end{array}$ & $\begin{array}{c}7 \\
21.2 \\
20.2 \\
21.9 \\
0.43\end{array}$ & $\begin{array}{c}9 \\
21.4 \\
19.7 \\
22.3 \\
0.62\end{array}$ & $\begin{array}{c}9 \\
21.4 \\
20.6 \\
21.9 \\
0.29\end{array}$ & $\begin{array}{l}15 \\
21.7 \\
19.4 \\
22.9 \\
0.48\end{array}$ & $\begin{array}{c}9 \\
21.6 \\
20.8 \\
23.1 \\
0.47\end{array}$ & $\begin{array}{l}16 \\
21.6 \\
20.9 \\
22.1 \\
0.20\end{array}$ & $\begin{array}{c}5 \\
22.5 \\
21.8 \\
22.9 \\
0.44\end{array}$ & $\begin{array}{l}11 \\
22.0 \\
21.3 \\
22.6 \\
0.24\end{array}$ & $\begin{array}{l}11 \\
21.3 \\
20.4 \\
22.1 \\
0.39\end{array}$ & $\begin{array}{c}3 \\
20.8 \\
20.0 \\
21.2 \\
0.77\end{array}$ & $\begin{array}{l}15 \\
20.9 \\
20.0 \\
22.1 \\
0.31\end{array}$ & $\begin{array}{l}30 \\
20.8 \\
19.5 \\
22.2 \\
0.24\end{array}$ & $\begin{array}{c}3 \\
18.8 \\
18.4 \\
19.5 \\
0.70\end{array}$ & $\stackrel{1}{18.9}$ \\
\hline \multicolumn{16}{|c|}{ Interorbital constriction } \\
\hline $\begin{array}{l}N \\
\text { Mean } \\
\text { Minimum } \\
\text { Maximum } \\
2 \text { sE }\end{array}$ & $\begin{array}{l}15 \\
13.2 \\
12.6 \\
13.5 \\
0.13\end{array}$ & $\begin{array}{c}7 \\
13.5 \\
12.9 \\
14.0 \\
0.28\end{array}$ & $\begin{array}{l}10 \\
13.1 \\
12.7 \\
13.7 \\
0.21\end{array}$ & $\begin{array}{c}9 \\
13.2 \\
12.5 \\
13.9 \\
0.29\end{array}$ & $\begin{array}{l}17 \\
13.5 \\
12.5 \\
14.3 \\
0.25\end{array}$ & $\begin{array}{c}9 \\
13.4 \\
13.1 \\
13.8 \\
0.15\end{array}$ & $\begin{array}{l}19 \\
13.2 \\
12.5 \\
14.0 \\
0.19\end{array}$ & $\begin{array}{c}6 \\
14.0 \\
13.4 \\
14.2 \\
0.26\end{array}$ & $\begin{array}{l}10 \\
14.0 \\
13.3 \\
14.6 \\
0.32\end{array}$ & $\begin{array}{l}13 \\
14.1 \\
13.2 \\
14.5 \\
0.20\end{array}$ & $\begin{array}{c}2 \\
13.1 \\
13.0 \\
13.1 \\
0.10\end{array}$ & $\begin{array}{l}12 \\
13.0 \\
12.3 \\
14.1 \\
0.31\end{array}$ & $\begin{array}{l}25 \\
12.6 \\
11.8 \\
13.4 \\
0.15\end{array}$ & $\begin{array}{c}3 \\
12.1 \\
11.7 \\
12.5 \\
0.47\end{array}$ & $\begin{array}{c}1 \\
11.2\end{array}$ \\
\hline \multicolumn{16}{|c|}{ Reflected red } \\
\hline $\begin{array}{l}N \\
\text { Mean } \\
\text { Minimum } \\
\text { Maximum } \\
\text { 2 SE }\end{array}$ & $\begin{array}{c}7 \\
15.6 \\
14.5 \\
17.0 \\
0.75\end{array}$ & $\begin{array}{c}6 \\
15.5 \\
14.0 \\
17.0 \\
0.97\end{array}$ & $\begin{array}{c}4 \\
14.6 \\
13.0 \\
17.0 \\
1.70\end{array}$ & $\begin{array}{c}8 \\
14.4 \\
11.5 \\
17.5 \\
1.33\end{array}$ & $\begin{array}{c}7 \\
16.9 \\
15.0 \\
21.0 \\
1.50\end{array}$ & $\begin{array}{c}9 \\
17.2 \\
15.0 \\
19.5 \\
0.91\end{array}$ & $\begin{array}{c}8 \\
18.2 \\
17.0 \\
20.0 \\
0.80\end{array}$ & $\begin{array}{c}6 \\
15.2 \\
14.0 \\
17.0 \\
0.92\end{array}$ & $\begin{array}{c}8 \\
16.3 \\
15.0 \\
18.5 \\
0.78\end{array}$ & $\begin{array}{l}11 \\
12.1 \\
10.5 \\
13.0 \\
0.40\end{array}$ & $\begin{array}{c}2 \\
17.3 \\
16.5 \\
18.0 \\
1.50\end{array}$ & $\begin{array}{l}13 \\
15.4 \\
12.5 \\
17.5 \\
0.88\end{array}$ & $\begin{array}{l}12 \\
14.1 \\
13.0 \\
16.0 \\
0.69\end{array}$ & $\begin{array}{l}2 \\
18.0 \\
17.0 \\
19.0 \\
2.00\end{array}$ & $\stackrel{1}{15.0}$ \\
\hline \multicolumn{16}{|c|}{ Reflected green } \\
\hline $\begin{array}{l}N \\
\text { Mean } \\
\text { Minimum } \\
\text { Maximum } \\
2 \text { SE }\end{array}$ & $\begin{array}{c}7 \\
9.4 \\
8.0 \\
12.0 \\
0.97\end{array}$ & $\begin{array}{l}6 \\
8.3 \\
7.0 \\
9.5 \\
0.72\end{array}$ & $\begin{array}{l}4 \\
8.0 \\
7.0 \\
9.0 \\
0.82\end{array}$ & $\begin{array}{c}8 \\
8.1 \\
6.5 \\
10.5 \\
1.04\end{array}$ & $\begin{array}{c}7 \\
9.2 \\
7.5 \\
12.0 \\
1.21\end{array}$ & $\begin{array}{c}9 \\
9.3 \\
8.5 \\
11.0 \\
0.60\end{array}$ & $\begin{array}{c}8 \\
9.3 \\
8.0 \\
10.5 \\
0.60\end{array}$ & $\begin{array}{c}6 \\
8.3 \\
7.0 \\
10.5 \\
1.17\end{array}$ & $\begin{array}{l}8 \\
8.1 \\
7.0 \\
9.0 \\
0.45\end{array}$ & $\begin{array}{c}11 \\
6.6 \\
6.0 \\
7.0 \\
0.24\end{array}$ & $\begin{array}{c}2 \\
9.5 \\
8.5 \\
10.5 \\
2.00\end{array}$ & $\begin{array}{c}13 \\
8.7 \\
7.0 \\
10.5 \\
0.62\end{array}$ & $\begin{array}{c}12 \\
8.1 \\
7.0 \\
10.5 \\
0.66\end{array}$ & $\begin{array}{c}2 \\
10.3 \\
9.5 \\
11.0 \\
1.50\end{array}$ & $\begin{array}{l}1 \\
8.0\end{array}$ \\
\hline \multicolumn{16}{|c|}{ Reflected blue } \\
\hline $\begin{array}{l}N \\
\text { Mean } \\
\text { Minimum } \\
\text { Maximum } \\
2 \text { SE }\end{array}$ & $\begin{array}{l}7 \\
7.5 \\
6.5 \\
8.5 \\
0.49\end{array}$ & $\begin{array}{l}6 \\
7.3 \\
6.0 \\
9.5 \\
0.99\end{array}$ & $\begin{array}{l}4 \\
6.4 \\
6.0 \\
7.0 \\
0.48\end{array}$ & $\begin{array}{l}8 \\
6.3 \\
5.0 \\
7.5 \\
0.63\end{array}$ & $\begin{array}{l}7 \\
7.6 \\
6.5 \\
9.5 \\
0.89\end{array}$ & $\begin{array}{l}9 \\
7.9 \\
7.5 \\
9.0 \\
0.39\end{array}$ & $\begin{array}{l}8 \\
7.6 \\
6.5 \\
8.5 \\
0.48\end{array}$ & $\begin{array}{l}6 \\
6.9 \\
6.5 \\
7.5 \\
0.40\end{array}$ & $\begin{array}{l}8 \\
6.8 \\
5.5 \\
7.5 \\
0.50\end{array}$ & $\begin{array}{l}11 \\
5.9 \\
4.5 \\
6.5 \\
0.38\end{array}$ & $\begin{array}{l}2 \\
8.0 \\
7.5 \\
8.5 \\
1.00\end{array}$ & $\begin{array}{c}13 \\
7.5 \\
6.5 \\
8.5 \\
0.44\end{array}$ & $\begin{array}{c}12 \\
6.9 \\
6.0 \\
10.5 \\
0.72\end{array}$ & $\begin{array}{l}2 \\
8.8 \\
8.5 \\
9.0 \\
0.50\end{array}$ & $\begin{array}{l}1 \\
7.5\end{array}$ \\
\hline
\end{tabular}


TABLE 4.-Results of four typical SS-STP analyses of geographic variation in southern banner-tailed kangaroo rats. Geographic origin of samples is shown in Fig. 2. Horizontal lines connect means of maximally nonsignificant subsets at the .05 level.

Length of hind foot

\begin{tabular}{lcccccccccccccc} 
Sample & 3 & 9 & 10 & 8 & 13 & 4 & 11 & 12 & 7 & 6 & 1 & 5 & 2 & 14 \\
Mean & 42.0 & 41.8 & 41.2 & 41.2 & 41.1 & 41.1 & 41.0 & 40.5 & 40.5 & 40.4 & 40.3 & 40.2 & 40.0 & 36.3 \\
\hline
\end{tabular}

Greatest length of skull

$\begin{array}{lcccccccccccccc}\text { Sample } & 11 & 12 & 13 & 9 & 5 & 10 & 3 & 7 & 8 & 6 & 4 & 2 & 1 & 14 \\ \text { Mean } & 37.5 & 37.4 & 37.3 & 37.2 & 36.5 & 36.5 & 36.5 & 36.4 & 36.4 & 36.3 & 36.2 & 35.8 & 35.5 & 34.1\end{array}$

Interorbital constriction

$\begin{array}{lllllllllllllll}\text { Sample } & 10 & 9 & 8 & 2 & 5 & 6 & 4 & 7 & 1 & 3 & 11 & 12 & 13 & 14\end{array}$

$\begin{array}{lllllllllllllll}\text { Mean } & 14.1 & 14.0 & 14.0 & 13.5 & 13.5 & 13.4 & 13.2 & 13.2 & 13.2 & 13.1 & 13.1 & 13.0 & 12.6 & 12.1\end{array}$

Color (red reflectance)

$\begin{array}{lllllllllllllll}\text { Sample } & 7 & 14 & 11 & 6 & 5 & 9 & 1 & 2 & 12 & 8 & 3 & 4 & 13 & 10\end{array}$ $\begin{array}{lllllllllllllll}\text { Mean } & 18.2 & 18.0 & 17.3 & 17.2 & 16.9 & 16.3 & 15.6 & 15.5 & 15.4 & 15.2 & 14.6 & 14.4 & 14.1 & 12.1\end{array}$

3 and 4, which are from relatively high elevations as compared with other samples from Jalisco and Zacatecas. Specimens from Tlaxcala (11) also had high values of reflected red. The two samples (12 and 13) that often grouped with sample 11 in external and cranial measurements fell among those with the low mean values for red reflectance. The sample from the vicinity of Mexico City (sample 10) had the lowest mean reading for red reflectance. The average value for this sample is a full 2 per cent less in reflectance than is the next lowest mean value ( sample 13).

Samples fell into three broadly overlapping, nonsignificant subsets for reflectance of green and four for reflectance of blue. They revealed approximately the same relationship as for reflected red. Sample 10 had a much lower mean than the other samples for green and blue, as it did for red.

\section{Multivariate Analysis}

Means for each sample for the three external and six cranial measurements and three color reflectance values were used in a NTSYS-multivariate analysis. Phenograms diagramming the phenetic relationships of southern banner-tailed kangaroo rats were computed by cluster analysis from both distance and cor- 


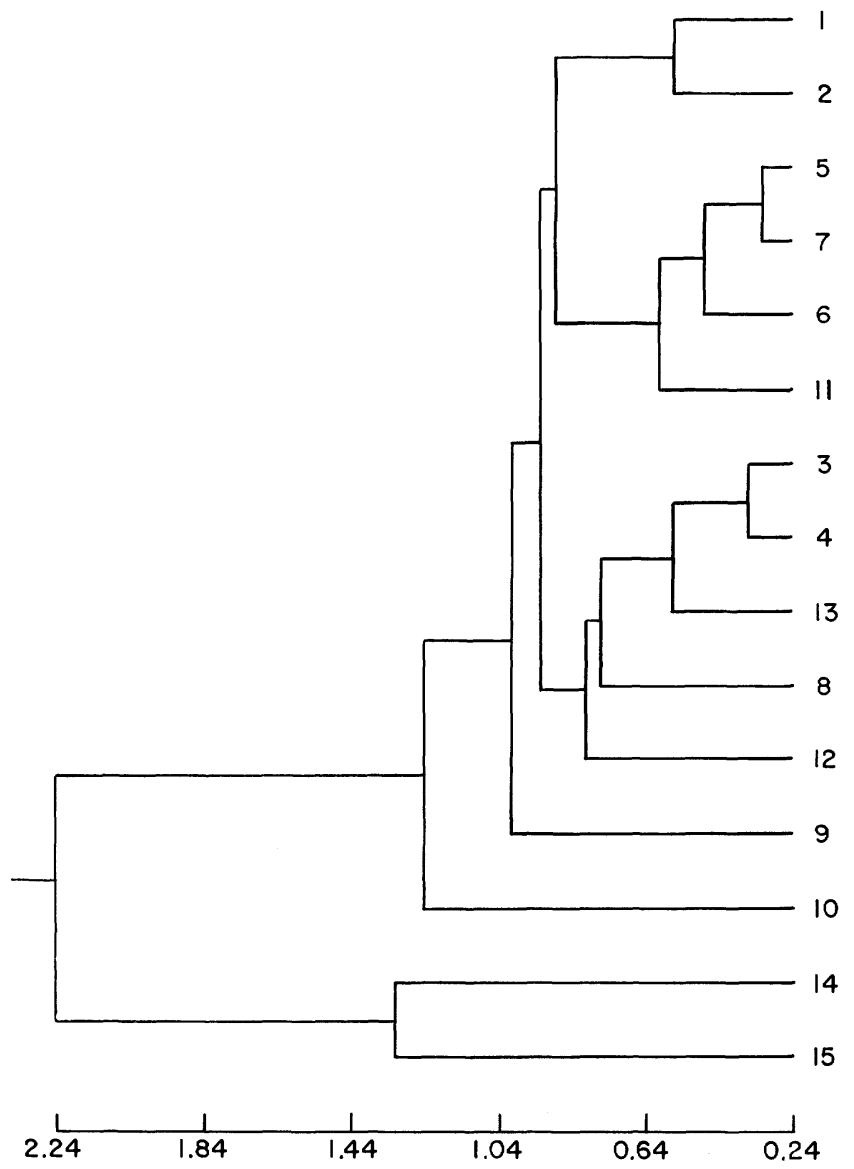

FIG. 1.-Distance phenogram resulting from cluster analysis of 15 geographic samples (see Fig. 2) of southern banner-tailed kangaroo rats.

relation matrices; the phenogram based upon the distance matrix is presented in Fig. 1. The samples in this phenogram are divided into two major clusters, one consisting of samples 14 and 15 and the second containing all others. Samples 14 and 15 are distantly separated in the first cluster. In the second cluster there are at least four major subclusters. Two of these consist of single samples 9 and 10 , but the other two contain five $(3,4,8,12$, and 13$)$ and six samples $(1,2,5,6,7$, and 11). The coefficient of cophenetic correlation for the distance phenogram was 0.931 .

Fig. 2 indicates the approximate areas from which the samples were drawn and the distance coefficient between the connected samples. In most cases, for ease of diagrammatic presentation, we have connected only adjacent samples. The largest distance coefficients were found between samples 13 and 14 (2.568) and between 13 and 15 (1.815). Distance coefficients of more than 


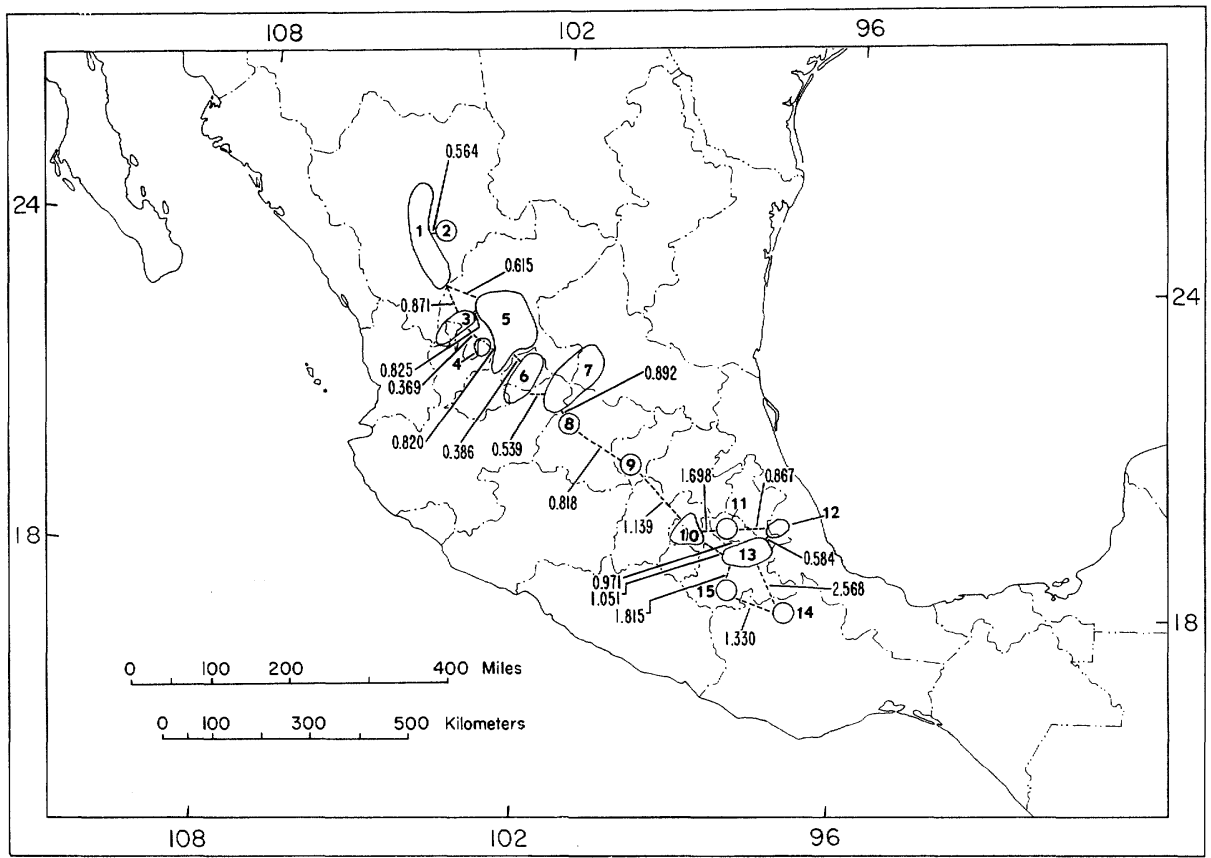

FIG. 2.-Map showing geographic location of 15 samples of southern banner-tailed kangaroo rats used for numerical analysis in this study, and distance coefficients between adjacent samples.

1.00 were generated between samples 9 and 10,10 and 11,10 and 13, and 14 and 15, and a coefficient of 0.971 was found between samples 11 and 13 . Distance coefficients of 0.8 to 0.9 were common, being found between six sets of localities.

The first three principal components were computed from the matrix of correlation among the 12 characters. The first principal component expresses 64.16 per cent of the phenetic variation, the second 19.09, and the third 6.30. Two-dimensional plots of the three principal components are shown in Fig. 3. From the results of the factor analysis (Table 5), it appears that both external and cranial size had a strong influence on the first component. With respect to positioning of samples along component $I$, the sample containing specimens that were smallest overall (14) is located on the far right; from that point, samples are arranged in ascending order relative to size, with the sample consisting of the largest individuals (sample 9) on the far left of the plot. Major factors in the second component were the color reflectance ratings, although depth of cranium and mastoid breadth had some influence. The positioning of samples along component II reveals that sample 10, which contained the darkest individuals, is near the top of the plot, and that samples 3 and 4, which also had low reflectance readings, occupy a somewhat lower 

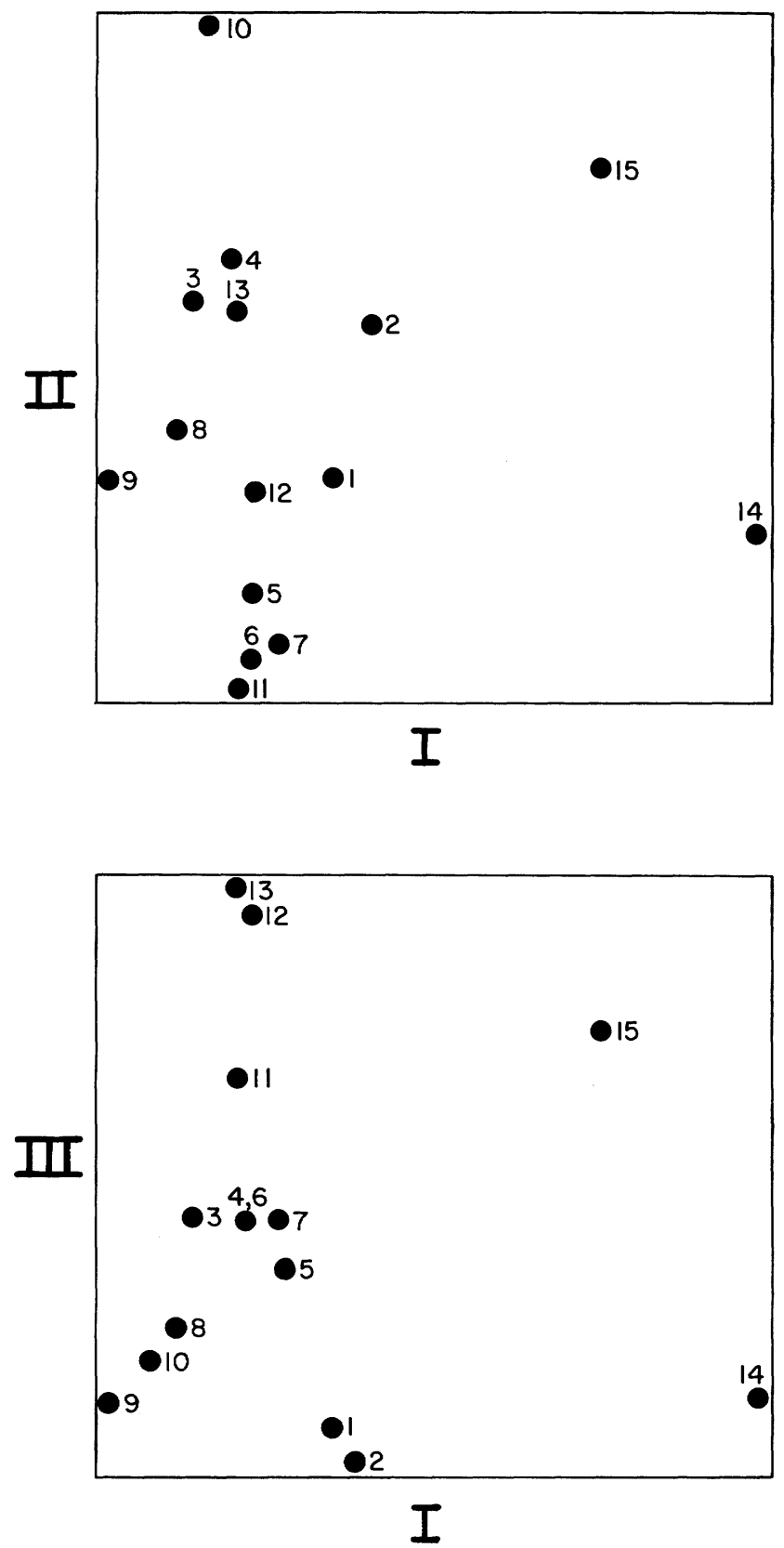

Fig. 3.-Two-dimensional projections of the first three principal components, illustrating the phenetic position of 15 samples of southern banner-tailed kangaroo rats. Top, component I plotted against component II; bottom, component I plotted against component III. 
TABLE 5.-Factor matrix from correlation among the 12 characters studied.

\begin{tabular}{lccc}
\hline \multicolumn{1}{c}{ Measurement } & $\begin{array}{c}\text { Factor } \\
\text { Component I }\end{array}$ & $\begin{array}{c}\text { Factor } \\
\text { Component II }\end{array}$ & $\begin{array}{c}\text { Factor } \\
\text { Component III }\end{array}$ \\
\hline Total length & -0.922 & -0.135 & 0.150 \\
Length of tail & -0.867 & -0.159 & -0.043 \\
Length of hind foot & -0.947 & -0.072 & -0.077 \\
Greatest length of skull & -0.848 & -0.163 & 0.423 \\
Length of maxillary toothrow & -0.891 & -0.109 & -0.224 \\
Depth of cranium & -0.867 & -0.246 & 0.195 \\
Mastoid breadth & -0.854 & -0.230 & 0.330 \\
Maxillary breadth & -0.891 & -0.137 & -0.277 \\
Interorbital constriction & -0.805 & -0.093 & -0.505 \\
Reflected red & 0.332 & -0.904 & -0.106 \\
Reflected green & 0.467 & -0.852 & -0.050 \\
Reflected blue & 0.659 & -0.720 & 0.065 \\
\hline
\end{tabular}

position on the component. Near the bottom of component II are samples that averaged high in reflectance readings. Factor analysis (Table 5) indicates that loading in the third component had high positive values for greatest length of skull and mastoid breadth and high negative values for maxillary breadth and interorbital constriction. The three samples (11, 12, and 13) that separate from the others in the third principal component are those that were shown in the univariate analysis to have among the largest means for mastoid breadth and among the smallest for maxillary breadth and interorbital constriction. The single specimen in sample 15 also appears to fall in the third component with samples 11,12 , and 13 , but it is widely separated from these samples in component $I$, which indicates that in overall size this specimen is much smaller than those in samples 11,12 , and 13.

\section{Bacular Morphology}

The bacula of Dipodomys ornatus (Lidicker, 1960b:496) and Dipodomys phillipsii (Burt, 1960:45) have been figured previously, but no comparison between them has been made. Burt (op. cit.) stated that the morphology of the single baculum from a Oaxacan specimen that he examined differed from all others in the genus in that the tip is upturned at a sharp angle from the shaft. This is not the case, however, in bacula of two specimens that we examined from México and Veracruz. These do have an upturned tip (Fig. 4 ), but the angle between the tip and shaft appears similar to that figured by Burt (1960:pl. 12) for other Dipodomys. The bacula of four specimens from within the previously understood range of ornatus (two from Jalisco, one each from Zacatecas and Guanajuato) agree morphologically with those figured by Lidicker (1960b:496) from Aguascalientes.

Little difference is evident in construction and morphology of the bacula of ornatus and phillipsii. That of ornatus is somewhat the larger, but it should be noted that the bacula of phillipsii we examined were from young adults, 

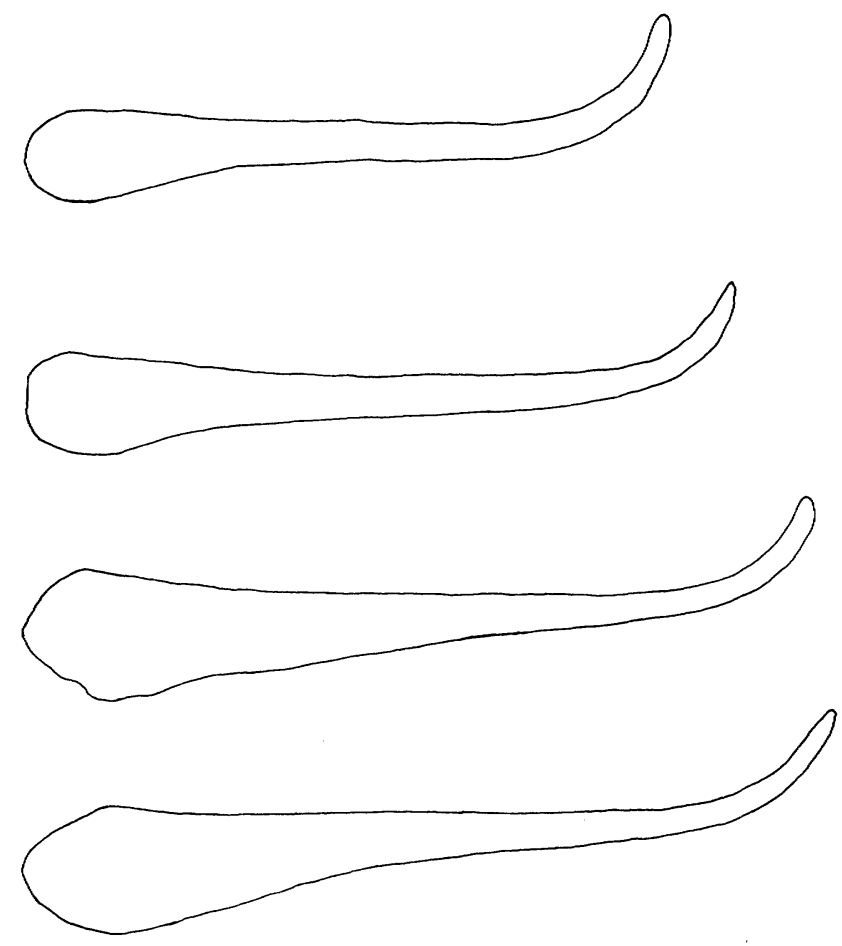

$1 \mathrm{~mm}$

FIG. 4.-Bacula of four southern banner-tailed kangaroo rats. Specimens represented (from top to bottom) are as follows: KU 19384, $2 \mathrm{~km} \mathrm{E} \mathrm{Perote,} \mathrm{Veracruz;} \mathrm{KU} \mathrm{48987,}$ 5 mi. S, 1 mi. W Texcoco, México; KU 48975, 8 mi. SE Zacatecas, Zacatecas; KU 48986, 4 mi. N, 5 mi. W León, Guanajuato.

because no other material was available. Bacular measurements are as follows (specimens from, respectively, México, Veracruz, Burt's rat from Oaxaca, Zacatecas, two from Jalisco, Guanajuato, mean and range of five bacula from Aguascalientes examined by Lidicker): length of baculum, 10.0, 9.1, 10.5, 11.3, $11.3,11.1,11.5,12.2$ (11.7-12.7); height of base, 1.3, 1.4, 1.3, 1.6, 2.0, 2.0, $1.4,1.8$ (1.4-2.2); width of base, 1.0, 0.9, 1.2, 1.6, 1.5, 1.6, 1.8, 1.6 (1.5-1.9).

\section{Taxonomic Conclusions}

We interpret the univariate and multivariate analyses as revealing that southern banner-tailed kangaroo rats represent one geographically variable species. The relatively minor cranial variations that Merriam (1894:110-111) used to characterize $D$. ornatus (such as a flatter cranium) are the result of individual and geographic variation within a population of this species. Therefore, in the following accounts all southern banner-tailed kangaroo rats are treated as a single species, Dipodomys phillipsii Gray. 
Within D. phillipsii, we recognize four subspecies. In the north, from Querétaro to central Durango, is Dipodomys phillipsii ornatus, which is characterized by medium to large size, relatively pale coloration, and medium to broad cranium. The nominate race, Dipodomys phillipsii phillipsii, is confined to the Valle de México and immediate vicinity; it is characterized by medium size, dark coloration, and broad interorbital region. Dipodomys phillipsii perotensis, which occurs in Tlaxcala, Puebla, and Veracruz, can be distinguished by large size, coloration intermediate between that of ornatus and phillipsii, a broad mastoid region, and narrow interorbital and maxillary regions. The fourth subspecies, Dipodomys phillipsii oaxacae, known from northern Oaxaca and southern Puebla, is much smaller than the others and pale in color.

\section{SYNOPSIS OF SUBSPECIES}

The four recognized subspecies of $D$. phillipsii are briefly described in the following accounts, and pertinent commentary is included on distribution and infrasubspecific variation. In the lists of specimens examined, localities in italic type are not plotted on the accompanying distribution map (Fig. 5) because crowded symbols would have resulted.

\section{Dipodomys phillipsii phillipsii Gray}

1841. Dipodomys phillipii [sic] Gray, Ann. Mag. Nat. Hist., ser. 1, 7:522 (see Coues, 1875:325, and Coues and Allen, 1877:540, for emendation of spelling). Type locality_" "near Real del Monte," Hidalgo.

Distribution.-Confined to Valle de México and immediately adjacent areas in Hidalgo, México, and the Distrito Federal (see Fig. 5).

Remarks.-The nominal subspecies is characterized by dark dorsal coloration, broad maxillary and interorbital regions relative to mastoid breadth, and in being medium for the species in general size. For comparison of $D$. p. phillipsii with other subspecies of the species, see accounts of those taxa.

According to Merriam (1893:84-86), after collecting a large series of D. phillipsii near Mexico City, E. W. Nelson attempted to obtain specimens in the vicinity of the type locality, Real del Monte, Hidalgo, at the extreme northern edge of the Valley of Mexico. His search in the vicinities of Real del Monte, Pachuca, Tula, San Agustín, and Irolo, all in Hidalgo, proved unsuccessful, although en route from Pachuca to Irolo, Nelson noted an area south of Pachuca that he believed might be suitable habitat for these kangaroo rats. Based on his failure to obtain specimens near the type locality, Nelson concluded that the locality recorded by Gray was erroneous and that the holotype most likely had originated from somewhere near Tlalpam, which was one of the important cities in the Valley of Mexico in the mid-1800's, and a place where D. phillipsii was abundant. Later, however, a specimen was obtained on 22 August 1942 (Davis, 1944:391) at a place $85 \mathrm{~km} \mathrm{~N}$ Mexico City (approximately $9 \mathrm{~km} \mathrm{~S}$ Pachuca, Hidalgo) casting considerable doubt on Nelson's conclusion. We believe it best to consider that the holotype of Dipodomys phillipsii came from the vicinity of Real del Monte, Hidalgo, at least until more convincing evidence to the contrary is available.

The one specimen examined from south of Pachuca (TCWC 3028) is a male with deciduous premolars and still in juvenile pelage. The pelage of this specimen is darker than in juveniles of ornatus, but paler than in juveniles of typical phillipsii. The possibility exists that specimens from this area represent intergrades between ornatus and phillipsii, but 


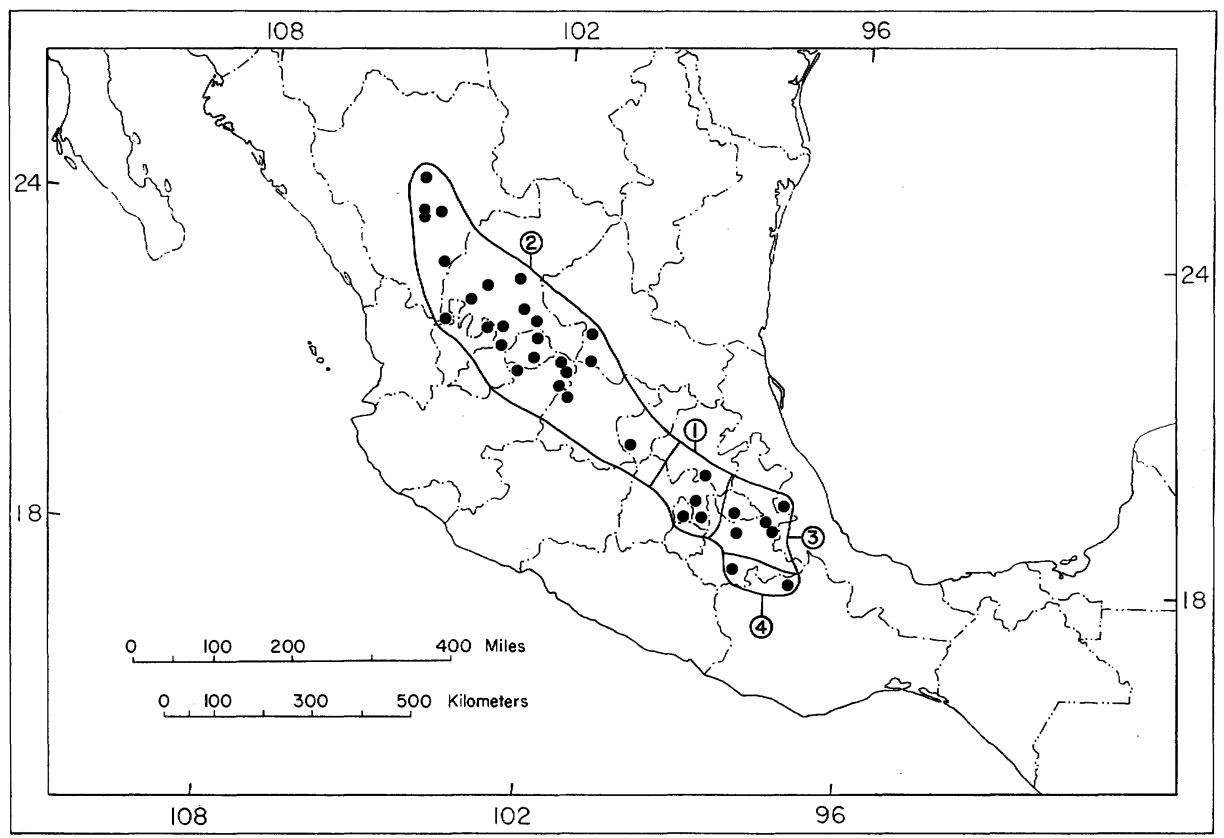

FIG. 5.-Distribution of subspecies of Dipodomys phillipsii: 1, D. p. phillipsii; 2, D. p. ornatus; 3, D. p. perotensis; 4, D. p. oaxacae.

adults are needed before any definitive statement can be made. One of us (Jones) did, however, examine the holotype (a badly preserved skin unaccompanied by skull) in the British Museum; its dorsal coloration is relatively dark, more or less typical of that found in specimens herein assigned to phillipsii.

Merriam (1893:86), quoting Nelson's field notes, stated that "they [D. phillipsii] were noted close to the peak of Huitzilac, near the Cruz del Marquez, at an altitude of 9000 feet." We have not seen specimens from this locality and it is not clear whether Nelson collected the rats there.

Specimens examined (38).-HIDALGO: near Real del Monte, 1 (BM-the holotype); $85 \mathrm{~km} \mathrm{~N}$ Mexico City, $8200 \mathrm{ft}, 1$ (TCWC). MEXICO: $5 \mathrm{mi} . \mathrm{S}, 1 \mathrm{mi}$. W Texcoco, $7350 \mathrm{ft}$, 1 (KU); $2 \mathrm{~km} \mathrm{~S} \mathrm{Huatongo,} 2700 \mathrm{~m}, 1$ (UNAM); Amecameca, 3 (USNM). DISTRITO FEDERAL: $17 \mathrm{~km}$ ESE Mexico City, 1 (TCWC) [labeled as in México, but reported as in the Distrito Federal by Villa-R., 1953:404, and Alvarez, 1961:409]; Cerro de la Caldera, $2300 \mathrm{~m}, 1$ (ENCB); Tlalpam, 26 (USNM); $\mathrm{km} 20$ de la cerretera México-Tláhuac, 1 (UNAM); Ajusco, 2 (USNM).

\section{Dipodomys phillipsii ornatus Merriam}

1894. Dipodomys ornatus Merriam, Proc. Biol. Soc. Washington, 9:110, 21 June. Type locality-Berriozábal, Zacatecas.

Distribution.-Recorded from the Mexican states of Durango, Zacatecas, Jalisco, Aguascalientes, San Luis Potosí, Guanajuato, and Querétaro. The northernmost record of occurrence is in the vicinity of Santa Cruz, Durango, and the southernmost is at Tequisquiapam, Querétaro (see Fig. 5).

Remarks.-This subspecies occupies the northern segment of the geographic range of the southern banner-tailed kangaroo rat. It is characterized by medium to large size and 
pale coloration. D. p. ornatus can be distinguished from $D$. p. phillipsii by its much paler color and relatively narrow interorbital region ( 57.9 to 60.9 per cent of mastoid breadth in nine samples of ornatus as compared with an average of 62.7 per cent in phillipsii). From D. p. perotensis, the subspecies ornatus differs in having a somewhat shorter skull on the average (greatest length in nine samples of ornatus ranged from 35.5 to 37.2 and in three of perotensis from 37.3 to 37.5 ), relatively broad maxillary and interorbital regions (maxillary breadth 94.2 to 96.6 per cent of mastoid breadth in ornatus and 89.3 to 90.8 in perotensis, interorbital breadth 57.9 to 60.9 per cent of mastoid breadth in ornatus and 54.8 to 57.2 in perotensis), and somewhat paler color. D. p. ornatus is easily distinguished from $D$. p. oaxacae by its much larger size.

A general increase in size in several cranial measurements was noted from north to south within the geographic range of ornatus. Samples from Durango generally had the smallest mean values, whereas those from Zacatecas, Aguascalientes, Jalisco, and San Luis Potosí were intermediate in size, and samples from Guanajuato and Querétaro had the largest mean values. Specimens from relatively high elevations (especially samples 3 and 4) average slightly darker in color than do those from lower areas.

Baker (1960:315-316) reported that individuals from the Guadiana lava fields (sample 2) were somewhat darker than typical specimens of ornatus. We find they are darker than rats from the vicinity of the type locality (sample 5), but that they are only slightly darker (revealed only in reflected green) than other specimens from Durango (sample 1), and that specimens from samples 3 and 4 are darker than those from the lava field in all three color readings taken (see Table 3 ).

Specimens from Tequisquiapam, Querétaro (9), show some tendencies toward $D . p$. phillipsii, but clearly are assignable to ornatus. They have the broadest interorbital region relative to mastoid breadth ( 60.9 per cent) of any sample of ornatus, and are somewhat darker than adjacent populations (as seen in reflectance readings of green and blue). Nevertheless, in all of these characters, the specimens from Querétaro resemble ornatus to a greater degree than phillipsii.

Alvarez (1961:409) cited from Dugés a record of Dipodomys phillipsii from San Diego de la Unión, Guanajuato. This record is of interest because it fills an otherwise rather broad gap in the known distribution of the species.

Specimens examined (141).-DURANGO: SE end Laguna de Santiaguillo, Santa Cruz, 4 (KU); 9 mi. N Durango, $6200 \mathrm{ft}, 1$ (KU); $6 \mathrm{mi}$. NW La Pila, $6150 \mathrm{ft}, 10$ (MSU); $4 \mathrm{mi}$. S Morcillo, $6450 \mathrm{ft}, 1$ (MSU); Durango, 4 (USNM); $16 \mathrm{mi}$. S, $20 \mathrm{mi}$. W Vicente Guerrero, $6675 \mathrm{ft}, 6$ (MSU). ZACATECAS: $12 \mathrm{mi}$. N, $7 \mathrm{mi}$. E Fresnillo, 4 (UNM); Laguna Valderama, $40 \mathrm{mi}$. W Fresnillo, $7800 \mathrm{ft}, 6$ (CAS); Valparaíso, 16 (USNM); Zacatecas, 4 (USNM); 2 mi. S, 5 mi. E Zacatecas, $7700 \mathrm{ft}, 1$ (MSU); 8 mi. SE Zacatecas, $7225 \mathrm{ft}, 4(\mathrm{KU})$; $2 \mathrm{mi}$. ESE Trancoso, $7000 \mathrm{ft}, 1$ (KU); Hda. San Juan Capistrano, 3 (USNM); Berriozábal, 2 (USNM); $2 \mathrm{mi}$. N Villanueva, $6500 \mathrm{ft}, 1$ (KU); Plateado, 5 (USNM). SAN LUIS POTOSI: $1 \mathrm{~km} \mathrm{~N}$ Arenal, 1 (LSU); 1 mi. W. Bledos, 1 (LSU); Bledos, 1 (LSU). JALISCO: La Mesa María de León, $7400 \mathrm{ft}, 14$ (KU); $10 \mathrm{mi}$. NW Matanzas, $7550 \mathrm{ft}, 5(\mathrm{KU}) ; 1 \mathrm{mi}$. NE Villa Hidalgo, $6550 \mathrm{ft}, 5(\mathrm{KU}) ; 5 \frac{1}{2} \mathrm{mi} . \mathrm{N}, 2 \mathrm{mi}$. W Guadalupe de Victoria, $7700 \mathrm{ft}, 1$ (MSU); $8 \mathrm{mi}$. W Encarnación de Díaz, $6000 \mathrm{ft}, 2$ (KU); $2 \mathrm{mi}$. SW Matanzas, $7550 \mathrm{ft}, 13$ (KU); Lagos, 1 (USNM). AGUASCALIENTES: 7 mi. $N$ Rincón de Romos, 1 (UNAM); 5 mi. NNE Rincón de Romos, 2 (KU); 3 mi. SW Aguascalientes, $6100 \mathrm{ft}, 1$ (KU). GUANAJUATO: $4 \mathrm{mi}$. N, $5 \mathrm{mi}$. W León, $7000 \mathrm{ft}, 8$ (KU). QUERETARO: Tequisquiapam, 12 (USNM).

\section{Dipodomys phillipsii perotensis Merriam}

1894. Dipodomys perotensis Merriam, Proc. Biol. Soc. Washington, 9:111, 21 June. Type locality-Perote, Veracruz.

1944. Dipodomys phillipsii perotensis, Davis, J. Mamm., 25:391, 21 December. 
Distribution.-Known from Tlaxcala, a limited area in west-central Veracruz in the vicinity of the type locality, and from eastern Puebla (see Fig. 5).

Remarks.-From D. p. phillipsii, the subspecies perotensis is distinguishable by its somewhat longer cranium (see Table 3), narrower maxillary breadth and interorbital constriction, and paler dorsal coloration. Comparisons of perotensis with other subspecies are in the accounts of those taxa. Specimens from Tlaxcala (sample 11) are paler than specimens in the other two samples of perotensis studied, but in other respects the three samples are fairly homogeneous.

Merriam (1893:86-88), quoting from the field notes of E. W. Nelson, stated that southern banner-tailed kangaroo rats were known from the northern and eastern base of Cerro de Malinche and from San Marcos, both places in Tlaxcala, and several localities in Puebla including Cañada Morelos, Esperanza, San Juan de los Llanos, and Ojo de Agua. We have not seen specimens from any of these localities and it is unclear (except for the lastmentioned place) from the account whether Nelson had specimens in hand or simply based his notes on field observations. Nelson did see a specimen from Ojo de Agua, Puebla, in a small collection at a college in the city of Puebla.

Specimens examined (67).-TLAXCALA: Huamantla, 3 (USNM). VERACRUZ: $2 \mathrm{~km}$ $\mathrm{N}$ Perote, $8000 \mathrm{ft}, 1$ (KU); $2 \mathrm{~km}$ W Perote, $8000 \mathrm{ft}, 1$ (KU); Perote, 7 (USNM); $2 \mathrm{~km}$ E Perote, $8300 \mathrm{ft}, 7$ (KU); Guadalupe Victoria (6 km SW Perote), $8300 \mathrm{ft}, 5$ (TCWC); $3 \mathrm{~km} W$ Limón, $7500 \mathrm{ft}, 3$ (KU); $2 \mathrm{~km}$ W Limón, $7500 \mathrm{ft}, 4$ (KU). PUEBLA: Laguna Salada (near Alchichia), $8000 \mathrm{ft}, 2$ (TCWC); $2 \mathrm{~km} \mathrm{~W}$ Atenco de Aljojuca, 1 (UNAM); $10 \mathrm{~km}$ W Chalchicomula, $8300 \mathrm{ft}, 1$ (TCWC); Chalchicomula, 31 (USNM); $7 \mathrm{mi}$. S, 3 mi. E Puebla, $6850 \mathrm{ft}, 1$ (KU).

\section{Dipodomys phillipsii oaxacae Hooper}

1947. Dipodomys phillipsii oaxacae Hooper, J. Mamm., 28:48, 17 February. Type localityTeotitlán, $950 \mathrm{~m}$, Oaxaca.

Distribution.-Known only from the type locality and one place in southern Puebla (see Fig. 5).

Remarks.-This subspecies is easily distinguished from all others of the species by its small size. Also, the color of oaxacae is much paler than that found in populations in adjacent areas of Puebla and Veracruz. Specimens we have examined exhibit the narrow maxillary ( 88.3 per cent) and interorbital ( 56.8 per cent) breadths relative to mastoid breadth that is characteristic also of $D$. p. perotensis.

D. p. oaxacae originally was described by Hooper (1947:48) on the basis of four specimens from Teotitlán, Oaxaca, until now the only known representatives of this distinctive subspecies. We have examined a specimen obtained by $R$. W. Dickerman at a place $1 \frac{1}{2}$ mi. W Tehuitzingo, Puebla, on 15 August 1954 that also appears assignable to oaxacae (this is the single individual in sample 15). This specimen is a young adult, but its appearance does not suggest that it ever will attain the size of the larger perotensis, which occurs to the northeast. We regard this specimen as only tentatively assigned to oaxacae until additional material becomes available from southern Puebla. It extends the known range of the subspecies approximately 130 kilometers to the west-northwest.

Specimens examined (5)._PUEBLA: $1 \frac{1}{2}$ mi. W Tehuitzingo, $3570 \mathrm{ft}, 1$ (KU). OAXACA: Teotitlán, $950 \mathrm{~m}, 4$ (UMMZ).

\section{Natural History}

\section{Habitat}

Although there has been no extensive ecological study of the southern banner-tailed kangaroo rat, notes on natural history of the species have 
appeared in several publications (Merriam, 1893:88-89; Davis, 1944:391; Villa-R., 1953:404; Dalquest, 1953:117; Baker and Greer, 1962:103; Hall and Dalquest, 1963:282-283). The accounts of Merriam and of Hall and Dalquest are especially noteworthy and both contain descriptions of the burrows of $D$. phillipsii. Most of the accounts record these kangaroo rats as commonest on sandy soils in areas of short grass where large clumps of prickly pear or nopal cactus and low thornbrush are found. It is interesting to compare the record given by Merriam (op. cit.) of E. W. Nelson's field accounts, written in 1892 and 1893, in which it was noted that the species was abundant in the vicinity of Tlalpam in the Valley of Mexico, and that given by Villa-R. (op. cit.), written in the early 1950's, in which it was stated that the species was scarce in the vicinity of Tlalpam; in fact, Villa was unable to obtain specimens from that area. Authors agree that this kangaroo rat is extremely difficult to trap, possibly accounting for Villa's inability to obtain specimens, but an alternative is that the species may have been displaced from the vicinity of Tlalpam by urbanization.

In the following paragraphs, we have given brief descriptions of seven representative localities at which $D$. phillipsii was obtained by field parties from the Museum of Natural History and for which field notes are available. These portray the situations in which this species may be found and list other species of mammals that may be expected to be found in association with the southern banner-tailed kangaroo rat.

$8 \mathrm{mi}$. SE Zacatecas, $7225 \mathrm{ft}$, Zacatecas.-R. H. Baker and a group of students visited this locality on 12-13 July 1952. Soils of the area are of volcanic origin and volcanic rocks were evident on the hills west of their campsite. Much of the land was under cultivation and many traps were placed along the edges of cornfields. Others were placed around clumps of grass and nopal cactus in a ravine near the camp. More than 190 mice were taken in 350 traps on the one night of trapping. Surprisingly, seven other species of heteromyid rodents were taken along with Dipodomys phillipsi-Perognathus flavus, P. hispidus, $P$. nelsoni, Dipodomys merriami, D. ordii, D. spectabilis, and Liomys irroratus. Other small mammals collected in this area included Thomomys umbrinus, Reithrodontomys fulvescens, R. megalotis, Peromyscus maniculatus, P. melanophrys, and Neotoma albigula.

La Mesa María de León, $7400 \mathrm{ft}$, Jalisco.-This locality, situated on a mesa approximately 1000 feet above the country immediately to the east, was visited from 21 to 24 June 1966 by P. L. Clifton and Genoways. The top of the mesa was a grassland supporting scattered oak trees, thus giving those areas not under cultivation a park-like appearance; the eastern edge of the mesa was steep and rock outcroppings were common there. Stands of trees and brush were much denser on the escarpment, with oak and manzanita being most abundant. Other species of mammals obtained at this place included Didelphis marsupialis, Sylvilagus floridanus, Lepus callotis, Spermophilus mexicanus, S. variegatus, Perognathus flavus, Peromyscus boylii, P. maniculatus, P. melanophrys, Sigmodon hispidus, Neotoma albigula, Urocyon cinereoargenteus, Spilogale putorius, and Mephitis macroura.

$10 \mathrm{mi}$. NW Matanzas, $7550 \mathrm{ft}$, Jalisco.-P. L. Clifton described the area northwest of Matanzas in mid-May 1966 as consisting of thousands of acres of unbroken grassland, with scattered patches of nopal cactus. Low stands of oaks grew on small hills scattered through the area. Other mammals collected were Sylvilagus floridanus, Lepus callotis, Spermophilus spilosoma, Thomomys umbrinus, Peromyscus difficilis, P. maniculatus, P. melanophrys, P. truei, Neotoma albigula, Canis latrans, and Spilogale putorius. 
TABLE 6.-Distribution by month of capture of 220 southern banner-tailed kangaroo rats of three age classes.

\begin{tabular}{|c|c|c|c|c|}
\hline Month & Juvenile & Young & Adult & Total \\
\hline January & - & - & - & - \\
\hline February & 1 & 1 & 1 & 3 \\
\hline March & - & - & - & - \\
\hline April & 0 & 1 & 30 & 31 \\
\hline May & 2 & 5 & 11 & 18 \\
\hline June & 2 & 4 & 33 & 39 \\
\hline July & 1 & 2 & 28 & 3.1 \\
\hline August & 0 & 1 & 6 & 7 \\
\hline September & 3 & 2 & 11 & 16 \\
\hline October & 1 & 1 & 16 & 18 \\
\hline November & 0 & 2 & 6 & 8 \\
\hline December & 15 & 10 & 24 & 49 \\
\hline
\end{tabular}

$2 \mathrm{mi}$. SW Matanzas, $7550 \mathrm{ft}$, Jalisco.-As the above locality, this place was essentially an unbroken prairie with scattered clumps of nopal cactus and thornbrush. Many traps were placed under clumps of nopal, which were surrounded by grass and weeds. The following species were obtained along with southern banner-tailed kangaroo rats on 13-14 October 1965: Spermophilus spilosoma, Thomomys umbrinus, Perognathus flavus, $P$. hispidus, Peromyscus difficilis, P. maniculatus, Onychomys torridus, Sigmodon fulviventer, and Mephitis macroura.

$8 \mathrm{mi}$. W Encarnación de Diaz, $6000 \mathrm{ft}$, Jalisco.-Vegetation in this part of Jalisco was primarily grassland, scattered with mesquite and other thorny bushes. Some cultivation (mostly corn) also prevailed. Traps were set along the edge of a cornfield and among weeds along a rock fence. Mammals obtained in the period 6 to 10 October 1965 included Sylvilagus audubonii, Spermophilus mexicanus, S. spilosoma, Perognathus flavus, P. hispidus, Dipodomys ordii, D. phillipsii, Reithrodontomys fulvescens, and Mus musculus.

$4 \mathrm{mi}$. N, $5 \mathrm{mi}$. W León, $7000 \mathrm{ft}$, Guanajuato.-This locality was visited by R. H. Baker and his field party shortly after they visited the locality in Zacatecas discussed above. The party camped on a grassy hillside where thornbrush and nopal cactus were abundant. There were numerous rock fences in the area along which many traps were set. Other rodents trapped at this locality included Perognathus flavus, P. hispidus, Reithrodontomys fulvescens, Peromyscus maniculatus, P. melanophrys, P. truei, and Baiomys taylori.

1/1/2 mi. W Tehuitzingo, $3570 \mathrm{ft}$, Puebla.-Vegetation west of Tehuitzingo was dominated by mesquite; some areas were under cultivation with the fields planted mainly to corn. R. W. Dickerman, a field representative of the Museum of Natural History, trapped on 15 August 1954 along a sandy river bed and among brush on the dry slope above the river. Aside from a single specimen of $D$. phillipsii, the only other small mammal obtained there was Liomys irroratus.

\section{Reproduction}

Of 27 adult female southern banner-tailed kangaroo rats that were examined for reproductive data, only three were found to contain embryos. A female taken on 1 June 1954 at the southeast end of Laguna de Santiaguillo, Durango, carried two embryos that measured 25 in crown-rump length, and two females obtained on 25 October 1950 at a place $1 \mathrm{mi}$. NE Villa Hidalgo, Jalisco, each contained three embryos that measured 16 (KU 40014) and 24 
(KU 40015) in crown-rump length. The remaining females, mostly collected in the months of June, July, and August, evinced no gross reproductive activity, and Hall and Dalquest (1963:283) reported that no females taken in Veracruz from late September to mid-November were pregnant. Length of testes for three adult males were 9 ( 3 June), 12 (23 June), and 11 (15 August).

Table 6 records 220 of the specimens we have examined by month of collection and by age. Only two months (January and March) are unrepresented by specimens. In only three months-April, August, and November-of the remaining 10 were no juveniles present in the sample and young individuals were present in all months. These data would seem to indicate a more prolonged reproductive period than can be deduced from the meager data on known reproduction in females.

\section{RESUMEN}

En un estudio de las ratas canguros del grupo Dipodomys phillipsii, se calcularon diversas variaciones geográficas y no geográficas. En la muestra proveniente de la vecindad de Perote, Veracruz, se reconocieron tres clases de edades (juveniles, subadultos, y adultos). En la muestra de Chalchicomula, Puebla, la variación estadística de los caracteres sexuales secundarios medidos fué poco significativa. Las variaciones geográficas de las medidas externas, craneales y del color del pelage fueron estudiados por medio de análisis de varianza y multivarianza para 15 muestras geográficas, las cuales revelaron que Dipodomys ornatus debe ser colocado como subespecie de D. phillipsii. Otras razas válidas son phillipsii, perotensis, y oaxacae.

Se incluye también algunas notas sobre la morfología del baculum, los lugares de vida y la reproducción de este grupo.

\section{Literature Cited}

Alvarez, T. 1961. Sinopsis de las especies Mexicanas del genero Dipodomys. Revista Soc. Mexicana Hist. Nat., 21:391-424.

Atchley, W. R. 1970. A biosystematic study of the subgenus Selfia of Culicoides (Diptera: Ceratopogonidae). Univ. Kansas Sci. Bull., 49:181-336.

Baker, R. H. 1960. Mammals of the Guadiana lava field Durango, Mexico. Publ. Mus., Michigan State Univ., Biol. Ser., 1:303-327.

Baker, R. H., AND J. K. Greer. 1962. Mammals of the Mexican state of Durango. Publ. Mus., Michigan State Univ., Biol. Ser., 2:25-154.

Burt, W. H. 1960. Bacula of North American mammals. Misc. Publ. Mus. Zool., Univ. Michigan, 113:1-76.

Chonte, J. R. 1970. Systematics and zoogeography of Middle American shrews of the genus Cryptotis. Univ. Kansas Publ., Mus. Nat. Hist., 19:195-317.

Coues, E. 1875. A critical review of the North American Saccomyidae. Proc. Acad. Nat. Sci. Philadelphia, pp. 272-327.

Coues, E., ANd J. A. Allen. 1877. Monographs of North American Rodentia. Bull. U. S. Geol. Surv. Territories, 11:xii $+\mathrm{x}+1-1091$.

Dalquest, W. W. 1953. Mammals of the Mexican state of San Luis Potosi. Louisiana State Univ. Studies, Biol. Sci. Ser., 1:1-229.

Davis, W. B. 1944. Notes on Mexican mammals. J. Mamm., 25:370-403. 
Dunnigan, P. B. 1967. Pocket gophers of the genus Thomomys of the Mexican state of Sinaloa. Radford Rev., 21:139-168.

Eisenberg, J. F. 1963. The behavior of heteromyid rodents. Univ. California Publ. Zool., 69:iv + 1-100.

Gray, J. E. 1841. A new genus of Mexican glirine Mammalia. Ann. Mag. Nat. Hist., ser. $1,7: 521-522$.

Hall, E. R., and F. H. Dale. 1939. Geographic races of the kangaroo rat, Dipodomys microps. Occas. Papers Mus. Zool., Louisiana State Univ., 4:47-62.

Hall, E. R., AND W. W. Dalquest. 1963. The mammals of Veracruz. Univ. Kansas Publ., Mus. Nat. Hist., 14:165-362.

Hooper, E. T. 1947. Notes on Mexican mammals. J. Mamm., 28:40-57.

-1952. A systematic review of the harvest mice (genus Reithrodontomys) of Latin America. Misc. Publ. Mus. Zool., Univ. Michigan, 77:1-255.

Lawlor, T. E. 1965. The Yucatan deer mouse, Peromyscus yucatanicus. Univ. Kansas Publ., Mus. Nat. Hist., 16:421-438.

Lidicker, W. Z., JR. 1960a. An analysis of intraspecific variation in the kangaroo rat Dipodomys merriami. Univ. California Publ. Zool., 67:125-218.

$1960 \mathrm{~b}$. The baculum of Dipodomys ornatus and its implication for superspecific groupings of kangaroo rats. J. Mamm., 41:495-499.

$\rightarrow$ LoNG, C. A. 1968. An analysis of patterns of variation in some representative Mammalia. Part I. A review of estimates of variability in selected measurements. Trans. Kansas Acad. Sci., 71:201-227.

1969. An analysis of patterns of variation in some representative Mammalia. Part II. Studies on the nature and correlation of measures of variation. Pp. 289-302, in Contributions in mammalogy ( J. K. Jones, Jr., ed.), Misc. Publ., Univ. Kansas Mus. Nat. Hist., 51:1-428.

Merriam, C. H. 1893. Rediscovery of the Mexican kangaroo rat, Dipodomys phillipsi Gray. Proc. Biol. Soc. Washington, 8:83-96.

- 1894. Preliminary descriptions of eleven new kangaroo rats of the genera Dipodomys and Perodipus. Proc. Biol. Soc. Washington, 9:109-115.

Power, D. M. 1970. Geographic variation of Red-winged Blackbirds in central North America. Univ. Kansas Publ., Mus. Nat. Hist., 19:1-83.

Rising, J. D. 1970. Morphological variation and evolution in some North American orioles. Syst. Zool., 19:315-351.

Schnell, G. D. 1970. A phenetic study of the suborder Lari (Aves). I. Methods and results of principal components analyses. Syst. Zool., 19:35-57.

SoKal, R. R., AND F. J. RoHLF. 1969. Biometry: the principles and practice of statistics in biological research. W. H. Freeman and Co., San Francisco, xiii +776 pp.

SpETH, R. L. 1969. Patterns and sequences of molts in the Great Basin pocket mouse, Perognathus parvus. J. Mamm., 50:284-290.

VillaA-R., B. 1953. Mamíferos silvestres del Valle de México. An. Inst. Biol. México, 23:269-492.

Museum of Natural History, The University of Kansas, Lawrence, 66044. Accepted 20 February 1971. 